\title{
Pequenos mamíferos não voadores em fragmentos de Mata Atlântica e áreas agrícolas em Viana, Espírito Santo, Brasil
}

\author{
Israel de Souza Pinto ${ }^{1,3}$, Ana Carolina Covre Loss², Aloísio Falqueto' \& Yuri Luiz Reis Leite \\ ${ }^{1}$ Laboratório de Parasitologia, Departamento de Patologia, Centro de Ciências da Saúde, \\ Universidade Federal do Espírito Santo - UFES, \\ Av. Marechal Campos 1468, Maruípe, CEP 29040-090, Vitória, ES, Brasil \\ ${ }^{2}$ Laboratório de Mastozoologia e Biogeografia, Departamento de Ciências Biológicas, \\ Centro de Ciências Humanas e Naturais, Universidade Federal do Espírito Santo - UFES, \\ Av. Marechal Campos, 1468, Maruípe, CEP 29043-900, Vitória, ES, Brasil \\ ${ }^{3}$ Autor para correspondência: Israel de Souza Pinto, e-mail: pintoisrael@gmail.com
}

PINTO, I.S., LOSS, A.C.C., FALQUETO, A. \& LEITE, Y.L.R. Non-flying small mammals in Atlantic Forest fragments and agricultural lands in Viana, state of Espírito Santo, Brazil. Biota Neotrop. 9(3): http://www. biotaneotropica.org.br/v9n3/en/abstract?inventory+bn03109032009

\begin{abstract}
We surveyed non-flying small mammals at Viana, Espírito Santo, southeastern Brazil, in 1981-1982 and 2006-2007. We trapped 439 non-flying small mammals belonging to three orders (Didelphimorphia, Rodentia, and Lagomorpha) and six families (Didelphidae, Sciuridade, Cricetidae, Muridae, Echimyidae, and Leporidae). The most abundant species were the rodents Akodon cursor and Nectomys squamipes and the marsupial Metachirus nudicaudatus. We recorded the echimyid rodent Euryzygomatomys spinosus for the first time in the state of Espírito Santo. The species richness $(S=21)$ and Shannon diversity index $(H=2.23)$ are among the highest recorded for Atlantic Forest small mammals in the state, even when compared to values from protected areas. These higher richness and diversity values are probably related to habitat heterogeneity and highlight the need for conservation of Viana's forest fragments, which are an important source of Atlantic Forest biodiversity.
\end{abstract}

Keywords: survey, Didelphimorphia, Lagomorpha, Rodentia.

PINTO, I.S., LOSS, A.C.C., FALQUETO, A. \& LEITE, Y.L.R. Pequenos mamíferos não voadores em fragmentos de Mata Atlântica e áreas agrícolas em Viana, Espírito Santo, Brasil. Biota Neotrop. 9(3): http:// www.biotaneotropica.org.br/v9n3/pt/abstract?inventory+bn03109032009

Resumo: Nós inventariamos os pequenos mamíferos não-voadores em Viana, Espírito Santo, sudeste do Brasil, em 1981-1982 e 2006-2007. Foram capturados 439 pequenos mamíferos não-voadores pertencentes a três ordens (Didelphimorphia, Rodentia e Lagomorpha) e seis famílias (Didelphidae, Sciuridade, Cricetidae, Muridae, Echimyidae e Leporidae). As espécies mais abundantes foram os roedores Akodon cursor e Nectomys squamipes e o marsupial Metachirus nudicaudatus. Registramos pela primeira vez a ocorrência do roedor equimídeo Euryzygomatomys spinosus no estado do Espírito Santo. A riqueza específica ( $\mathrm{S}=21)$ e o índice de diversidade de Shannon $(H=2,23)$ estão entre os maiores registrados para pequenos mamíferos da Mata Atlântica no estado, mesmo quando comparados aos valores obtidos para unidades de conservação. Esses maiores valores de riqueza e diversidade encontrados estão provavelmente associados à heterogeneidade de hábitat e evidenciam a necessidade de conservação dos fragmentos florestais de Viana, os quais são importantes depositários da biodiversidade da Mata Atlântica.

Palavras-chave: inventário, Didelphimorphia, Lagomorpha, Rodentia. 


\section{Introdução}

O bioma Mata Atlântica é considerado um dos locais de maior biodiversidade do mundo, albergando cerca de $7 \%$ das espécies que ocorrem no planeta (Myers et al. 2000, Quintela 1990), estando também entre as mais ameaçadas florestas tropicais (Mittermeier et al. 1998) devido aos altos níveis de degradação e de fragmentação de seus ambientes naturais (Law \& Dickman 1998). Tanto a degradação quanto a fragmentação do hábitat modificam substancialmente a estrutura das populações de animais silvestres, podendo inclusive afetar a riqueza e a diversidade de espécies (Law \& Dickman 1998). A destruição da Mata Atlântica foi ocasionada principalmente pela ocupação humana desordenada da região litorânea do Brasil. Inicialmente, as florestas nativas foram destruídas pelo extrativismo vegetal $\mathrm{e}$, posteriormente, para dar lugar à agropecuária e às grandes cidades. Com isso, a Mata Atlântica cobre atualmente menos de 10\% da área que ocupava antes da chegada dos colonizadores portugueses ao Brasil em 1500 (Dean 1995). O estado do Espírito Santo apresenta apenas $8 \%$ do território ainda coberto por formações vegetais de Mata Atlântica, sendo que essa formação cobria cerca de $90 \%$ do território capixaba antes da colonização (IPEMA 2005). Dessa atual porcentagem, a maior parte das áreas é representada por unidades de conservação pequenas ou por pequenos fragmentos florestais em propriedades privadas.

Atualmente, a Mata Atlântica abriga cerca de 250 espécies de mamíferos, sendo 69 endêmicas. Didelphimorphia e Rodentia são as ordens mais diversas de mamíferos não-voadores desse bioma (Fonseca et al. 1996), com pelo menos 23 e 79 espécies, respectivamente, correspondendo a mais da metade da diversidade de mamíferos neotropicais. Muitos mamíferos são dispersores de sementes, desempenhando importante papel ecológico dentro dos ecossistemas. Além disso, são de grande interesse para a saúde humana, pois podem atuar como reservatórios de parasitas que causam doenças de origem zoonótica no homem. Levantamentos faunísticos permitem uma avaliação da distribuição da biodiversidade, o que contribui para estudos de taxonomia e biogeografia. Tais estudos elucidam os padrões e processos responsáveis pela evolução e diversificação da fauna, sendo imprescindíveis para definir estratégias de manejo e conservação da vida silvestre, além de auxiliarem na compreensão de ciclos de transmissão de zoonoses, uma vez que identificam as áreas de ocorrência dos animais potencialmente reservatórios.

No estado do Espírito Santo, a mastofauna ainda é pouco estudada, principalmente no que diz respeito à sua distribuição, sendo que a maioria das pesquisas é direcionada às unidades de conservação (Ruschi et al. 1978, Paresque et al. 2004, Moreira et al. 2008). Baseados em registros de museus e literatura científica, Moreira et al. (2008) confeccionaram uma lista de mamíferos silvestres que ocorrem no Espírito Santo e identificaram as regiões que são lacunas de conhecimento da mastofauna do estado. Nesse estudo, o município de Viana foi categorizado como uma das áreas que necessita de mais estudos de levantamento de espécies de mamíferos devido à carência de registros. Ainda nesse município, são constantes os casos de leishmaniose tegumentar americana (LTA) e pequenos mamíferos podem atuar como reservatórios do agente etiológico da doença (Falqueto \& Ferreira 2005).

O presente trabalho teve como objetivo inventariar a fauna de pequenos mamíferos não-voadores em fragmentos de Mata Atlântica no município de Viana, estado do Espírito Santo.

\section{Material e Métodos}

O estudo foi desenvolvido no município de Viana, que dista aproximadamente $15 \mathrm{~km}$ da cidade de Vitória, capital do estado do Espírito Santo, fazendo parte da região metropolitana da Grande Vitória. O clima é tropical, com pluviosidade máxima de dezembro a fevereiro e período seco de julho a agosto. É uma região muito acidentada em alguns trechos, constituída de serras cujos morros determinam a formação de vales estreitos, quentes e úmidos. Segundo Ballestrero (1951), o predomínio fito-fisionômico era o de Mata Atlântica, que foi parcialmente substituída por cafezais e esses, por sua vez, foram sendo gradativamente substituídos por bananais após 1945 . O município de Viana não possui qualquer unidade de conservação e todos os fragmentos florestais estão localizados em propriedades particulares. Embora pequenos, existe um número considerável de fragmentos no município os quais foram preservados possivelmente por estarem localizados em áreas impróprias à agropecuária devido ao relevo acidentado e ao solo pedregoso (Feitoza 1999). Tais remanescentes florestais podem ser considerados depositários de boa parte da biodiversidade nativa da região.

A amostragem foi realizada em dois períodos: o primeiro de novembro de 1981 a outubro de 1982 e o segundo de maio de 2006 a dezembro de 2007. As coletas foram feitas em fragmentos florestais e bananais das localidades de Coacas (20 21' $33^{\prime \prime} \mathrm{S}$ e $\left.40^{\circ} 28^{\prime} 22^{\prime \prime} \mathrm{W}\right)$,

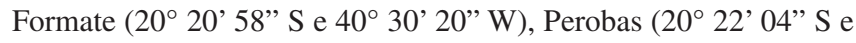
$40^{\circ} 28^{\prime} 31^{\prime \prime}$ W) e Povoação (2022' 44" S e 40 28' 31' W) (Figura 1) durante 60 dias no primeiro período, sendo utilizadas 21 armadilhas de isca do tipo gaiola de arame galvanizado $(32 \times 15 \times 15 \mathrm{~cm})$ estabelecidas em transectos, com espaçamento mínimo de 10 metros entre elas. No segundo período, foram amostrados durante 45 dias os remanescentes florestais e bananais das localidades de Fazenda Boa Baixa $\left(20^{\circ} 23^{\prime} 12^{\prime}\right.$ 'S e $40^{\circ} 27^{\prime} 25^{\prime}$ ' W), Formate, Pimenta $\left(20^{\circ} 22^{\prime} 45^{\prime}\right.$ 'S e $\left.40^{\circ} 28^{\prime} 06^{\prime \prime} \mathrm{W}\right)$ com 21 armadilhas de isca do tipo gaiola de arame galvanizado e das localidades de Coacas e Ribeira ( $20^{\circ} 23^{\prime} 12^{\prime \prime} \mathrm{S}$ e $\left.40^{\circ} 27^{\prime} 24^{\prime \prime} \mathrm{W}\right)$ com 20 armadilhas de isca do tipo Sherman de alumínio $(30 \mathrm{~cm} \times 9,5 \mathrm{~cm} \times 8,0 \mathrm{~cm})$ (Figura 1). Todas as armadilhas foram alocadas no solo no primeiro período, enquanto no segundo período foram alocadas 15 armadilhas no solo e 5 em galhos de árvores, a cerca de 1-2 m de altura em relação ao solo. $\mathrm{O}$ esforço amostral foi de 1260 armadilhas-noite no primeiro período e 900 armadilhas-noite no segundo período, sendo 675 armadilhas-noite no solo e 225 armadilhas-noite em galhos de árvore. As armadilhas foram iscadas com banana e verificadas a cada manhã. No primeiro período de amostragem, a maioria dos espécimes capturados foi identificada e solta, sendo que pelo menos um indivíduo por espécie de cada localidade foi coletado (Anexo I). No segundo período, todos os indivíduos capturados foram coletados e taxidermizados. A taxonomia seguiu Wilson \& Reeder (2005), com modificações de Oliveira \& Bonvicino (2006), e os espécimes testemunho dos mamíferos foram depositados na Coleção de Vertebrados da Universidade Federal do Espírito Santo (UFES). Aqueles que ainda não foram tombados estão identificados pelas inicias dos coletores e números de campo, sendo: Valéria Fagundes (inicial LGA), Leonora Pires Costa (LPC) e Simone Lóss de Freitas (SLF) (Anexo I).

Foram calculados a riqueza específica (S) e os índices de equitabilidade (J) e de diversidade de Shannon (H). Também foi calculada a constância de ocorrência das espécies pela fórmula de Dajoz (1973): $\mathrm{C}=\mathrm{P} \times 100 / \mathrm{N}$, em que $\mathrm{P}=$ número de capturas em que apareceu a espécie e $\mathrm{N}$ = número de capturas realizadas. De acordo com os dados de constância, formaram-se as seguintes categorias para as espécies: constantes - presentes em mais de 50\% das amostras; acessórias presentes entre 25 e $50 \%$ das amostras; ocasionais - presentes em menos de $25 \%$ das amostras.

\section{Resultados}

Foram capturados 439 pequenos mamíferos não voadores $(\mathrm{S}=21$; $\mathrm{H}=2$,228), sendo 252 no primeiro período, representando $20 \%$ de 


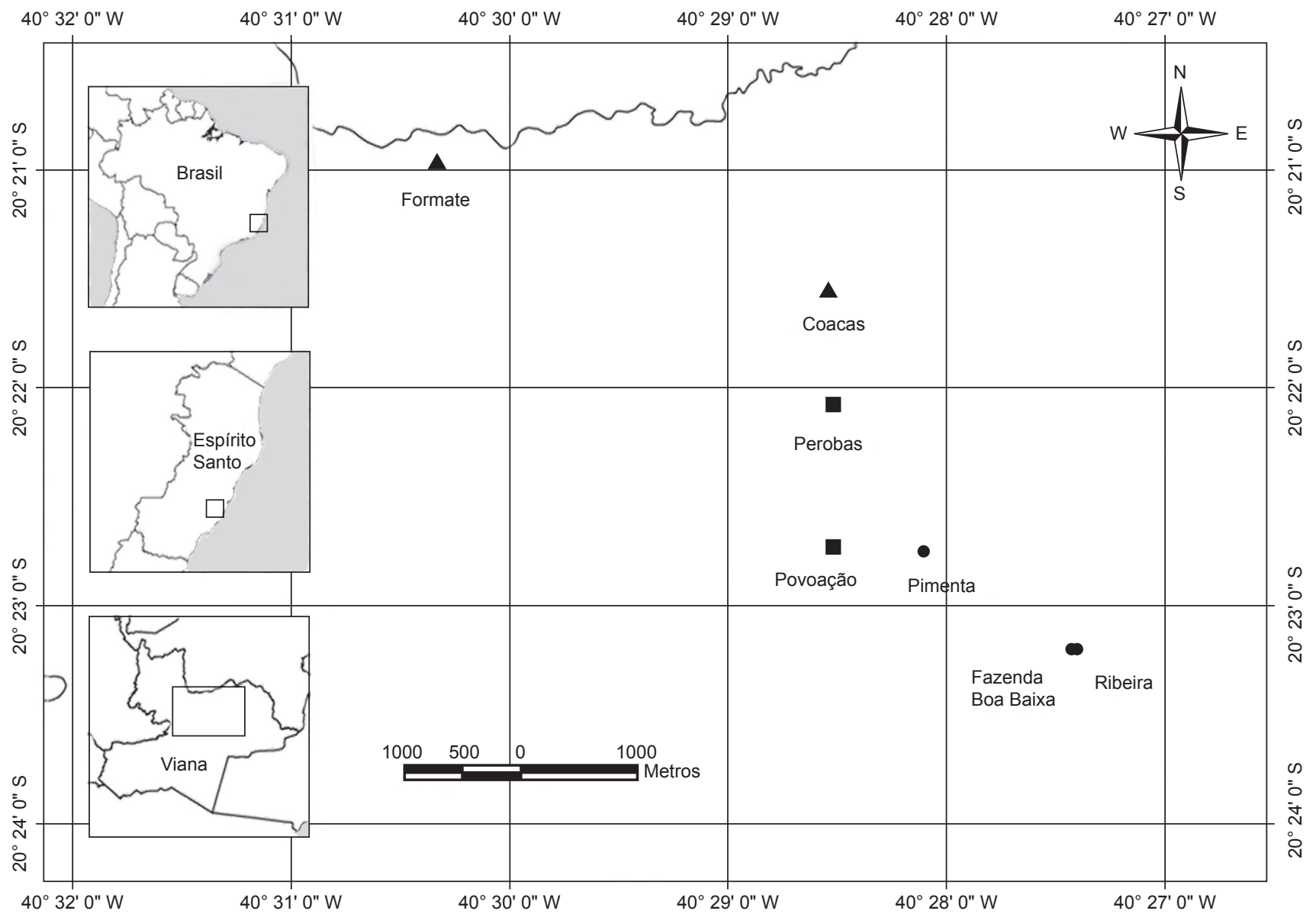

Figura 1. Mapa das localidades de coleta de pequenos mamíferos não voadores em Viana, Espírito Santo, Brasil. Os quadrados indicam áreas amostradas no período de setembro de 1981 a outubro de 1982, os círculos indicam as localidades amostradas de maio de 2006 a dezembro de 2007 e os triângulos indicam as localidades amostradas em ambos os períodos.

Figure 1. Map showing collecting localities of non-flying small mammals at Viana, Espírito Santo, Brazil. Squares represent areas sampled from September 1981 to October 1982, circles indicate localities sampled from May 2006 to December 2007, and triangles indicate localities sampled in both periods.

sucesso de captura, e 187 no segundo, representado $20,8 \%$ de sucesso de captura. Foram registradas 21 espécies distribuídas em seis famílias e três ordens (Tabela 1), o que corresponde a $47 \%$ do total de espécies de pequenos mamíferos não voadores registradas no estado do Espírito Santo (segundo Moreira et al. 2008) e 24\% da Mata Atlântica (segundo Fonseca et al. 1996). A localidade de Coacas apresentou a maior riqueza específica, seguida por Formate, enquanto esta apresentou o maior índice de diversidade, seguida por Pimenta (Tabela 1). A maior riqueza específica foi registrada para a família Didelphidae, seguida pela família Cricetidae. As espécies mais abundantes foram Akodon cursor, Nectomys squamipes e Metachirus nudicaudatus.

Quanto à constância de ocorrência: Marmosa murina, M. nudicaudatus, A. cursor, N. squamipes e Trinomys paratus foram consideradas espécies constantes; Didelphis aurita, Marmosops incanus e Micoureus paraguayanus foram consideradas acessórias; sendo as demais consideradas ocasionais.

\section{Discussão}

A diversidade de pequenos mamíferos encontrada no município de Viana $(\mathrm{H}=2,23)$ foi maior que a encontrada por Bonvicino et al. (2002) em área de Mata Atlântica com vegetação alterada $(H=2,04)$, superior ao maior índice encontrado por Passamani (2003) em áreas de Mata Atlântica no município de Santa Teresa $(H=2,02)$ e maior que a encontrada por Carão (2008) utilizando armadilhas de isca e fojos ("pitfalls") na Reserva Biológica de Duas Bocas (RBDB) no município de Cariacica $(H=2,16)$, que fica a menos de $10 \mathrm{~km}$ em linha reta da nossa área de estudo. A diversidade na RBDB é menor que a de Viana mesmo quando levadas em consideração somente capturas efetuadas em armadilhas de isca ( $\mathrm{H}=1,63$; Carão, 2008). No mesmo sentido, a riqueza de espécies no município de Viana $(\mathrm{S}=21)$ também foi maior que a encontrada por Passamani (2003) $(\mathrm{S}=20)$ e Carão (2008) ( $\mathrm{S}=17)$. As áreas de Mata Atlântica de Santa Teresa e da RBDB em Cariacica são reconhecidas por apresentarem grande diversidade de pequenos mamíferos (Moreira et al. 2008).

No presente trabalho, registramos pela primeira vez a ocorrência do roedor equimídeo Euryzygomatomys spinosus no estado do Espírito Santo. Ela já havia sido registrada na porção de Minas Gerais do Parque Nacional do Caparaó (Bonvicino et al. 1997), bem próximo da divisa com o Espírito Santo. Essa espécie inclusive não consta da lista dos mamíferos do estado publicada por Moreira et al. (2008). Nós coletamos um exemplar da espécie na localidade de Coacas e verificamos que existem outros exemplares depositados no Museu Nacional, Universidade Federal do Rio de Janeiro, coletados no município de Santa Teresa na década de 1940. Esses registros no estado 
Tabela 1. Espécies, número de indivíduos, riqueza específica (S), índice de equitabilidade (J) e índice de diversidade de Shannon (H) de pequenos mamíferos não voadores capturados de setembro de 1981 a outubro de 1982 (A) e de maio de 2006 a dezembro de 2007 (B), em cada localidade no município de Viana, Espírito Santo, Brasil.

Table 1. Species, number of specimens, species richness (S), equitability index ( $\mathrm{J})$ and Shannon's diversity index $(\mathrm{H})$ of non-flying small mammals trapped from September 1981 to October 1982 (A) and from May 2006 to December 2007 (B) at each locality in Viana municipal district, Espírito Santo, Brazil.

\begin{tabular}{|c|c|c|c|c|c|c|c|c|c|c|c|c|c|c|c|c|c|}
\hline \multirow[t]{2}{*}{ Espécies } & \multicolumn{2}{|c|}{ Coacas } & \multicolumn{2}{|c|}{$\begin{array}{l}\text { Faz. Boa } \\
\text { Baixa }\end{array}$} & \multicolumn{2}{|c|}{ Formate } & \multicolumn{2}{|c|}{ Perobas } & \multicolumn{2}{|c|}{ Pimenta } & \multicolumn{2}{|c|}{ Povoação } & \multicolumn{2}{|c|}{ Ribeira } & \multicolumn{2}{|c|}{ Sub-total } & \multirow[t]{2}{*}{ Total } \\
\hline & $\mathbf{A}$ & B & $\mathbf{A}$ & B & $\mathbf{A}$ & $\mathbf{B}$ & $\mathbf{A}$ & $\mathbf{B}$ & $\mathbf{A}$ & $\mathbf{B}$ & $\mathbf{A}$ & B & $\mathbf{A}$ & B & $\mathbf{A}$ & B & \\
\hline \multicolumn{18}{|l|}{ DIDELPHIMORPHIA } \\
\hline \multicolumn{18}{|l|}{ Didelphidae } \\
\hline $\begin{array}{l}\text { Caluromys philander } \\
\text { (Linnaeus, 1758) }\end{array}$ & - & - & - & 1 & - & - & - & - & - & 2 & - & - & - & - & - & 3 & 3 \\
\hline $\begin{array}{l}\text { Didelphis aurita } \\
\text { (Wied-Neuwied, 1826) }\end{array}$ & 3 & 1 & - & 3 & 3 & - & 7 & - & - & 6 & 4 & - & - & 3 & 17 & 13 & 30 \\
\hline $\begin{array}{l}\text { Gracilinanus microtarsus } \\
\text { (Wagner, 1842) }\end{array}$ & - & - & - & - & - & - & - & - & - & 1 & - & - & - & 1 & - & 2 & 2 \\
\hline $\begin{array}{l}\text { Marmosa murina } \\
\text { (Linnaeus, 1758) }\end{array}$ & 2 & 6 & - & 10 & 5 & 3 & 1 & - & - & 4 & 1 & - & - & 1 & 9 & 24 & 33 \\
\hline Marmosops incanus (Lund, 1840) & - & & - & - & 3 & - & 3 & - & - & 6 & 4 & - & - & 4 & 10 & 10 & 20 \\
\hline $\begin{array}{l}\text { Metachirus nudicaudatus } \\
\text { (Desmarest, 1817) }\end{array}$ & 9 & 2 & - & 5 & 8 & - & 4 & - & - & 7 & 13 & - & - & 3 & 34 & 17 & 51 \\
\hline $\begin{array}{l}\text { Micoureus paraguayanus } \\
\text { (Tate, 1931) }\end{array}$ & - & 3 & - & 2 & 2 & - & 1 & - & - & 6 & - & - & - & 5 & 3 & 16 & 19 \\
\hline $\begin{array}{l}\text { Monodelphis americana } \\
\text { (Müller, 1776) }\end{array}$ & 1 & - & - & 1 & - & - & - & - & - & - & 1 & - & - & - & 2 & 1 & 3 \\
\hline Philander frenatus (Olfers, 1818) & 2 & - & - & - & - & - & - & - & - & - & 1 & - & - & - & 3 & - & 3 \\
\hline \multicolumn{18}{|l|}{ RODENTIA } \\
\hline \multicolumn{18}{|l|}{ Sciuridae } \\
\hline $\begin{array}{l}\text { Guerlinguetus ingrami } \\
\text { (Thomas, 1901) }\end{array}$ & - & - & - & - & - & - & - & - & - & 5 & - & - & - & 1 & - & 6 & 6 \\
\hline \multicolumn{18}{|l|}{ Cricetidae } \\
\hline Akodon cursor (Winge, 1887) & 25 & 30 & - & 21 & 15 & - & 2 & - & - & 1 & 10 & - & - & 1 & 52 & 53 & 105 \\
\hline $\begin{array}{l}\text { Nectomys squamipes } \\
\text { (Brants, 1827) }\end{array}$ & 9 & 2 & - & 4 & 16 & 4 & 9 & - & - & 12 & 44 & - & - & - & 78 & 22 & 100 \\
\hline $\begin{array}{l}\text { Oecomys catherinae } \\
\text { Thomas, } 1909\end{array}$ & - & - & - & 3 & - & - & - & - & - & - & - & - & - & - & - & 3 & 3 \\
\hline $\begin{array}{l}\text { Oligoryzomys nigripes } \\
\text { (Olfers, 1818) }\end{array}$ & 1 & - & - & - & - & - & - & - & - & - & - & - & - & - & 1 & - & 1 \\
\hline $\begin{array}{l}\text { Oxymycterus dasytrichus } \\
\text { (Schinz, 1821) }\end{array}$ & 1 & - & - & - & 4 & - & 1 & - & - & - & 1 & - & - & - & 7 & - & 7 \\
\hline $\begin{array}{l}\text { Rhipidomys mastacalis } \\
\text { (Lund, 1840) }\end{array}$ & - & - & - & - & - & - & - & - & - & - & - & - & - & 1 & - & 1 & 1 \\
\hline \multicolumn{18}{|l|}{ Muridae } \\
\hline Rattus rattus (Linnaeus, 1758) & 1 & - & - & - & 1 & - & 2 & - & - & - & 1 & - & - & - & 5 & - & 5 \\
\hline \multicolumn{18}{|l|}{ Echimyidae } \\
\hline $\begin{array}{l}\text { Euryzygomatomys spinosus } \\
\text { (Fischer, 1814) }\end{array}$ & 1 & - & - & - & - & - & - & - & - & - & - & - & - & - & 1 & - & 1 \\
\hline $\begin{array}{l}\text { Phyllomys pattoni } \\
\text { Emmons et al., } 2002\end{array}$ & 1 & - & - & - & - & - & - & - & - & - & - & - & - & - & 1 & - & 1 \\
\hline Trinomys paratus (Moojen, 1948) & 1 & - & - & 6 & 15 & 3 & 10 & - & - & 5 & 1 & - & - & 2 & 27 & 16 & 43 \\
\hline \multicolumn{18}{|l|}{ LAGOMORPHA } \\
\hline \multicolumn{18}{|l|}{ Leporidae } \\
\hline $\begin{array}{l}\text { Sylvilagus brasiliensis } \\
\text { (Linnaeus, 1758) }\end{array}$ & 1 & - & - & - & 1 & - & - & - & - & - & - & - & - & - & 2 & - & 2 \\
\hline Total & 58 & 44 & & 56 & 73 & 10 & 40 & & & 55 & 81 & & & 22 & 252 & 187 & 439 \\
\hline S & & 5 & & 0 & & & 1 & & 1 & & 1 & & 1 & & 16 & 14 & 21 \\
\hline $\mathrm{J}$ & & 21 & & 23 & & & 0,8 & & 0,9 & & 0,6 & & 0,9 & & 0,740 & 0,833 & 0,731 \\
\hline $\mathrm{H}$ & & 84 & & 95 & & & 1,9 & & 2,2 & & 1,5 & & 2,1 & & 2,053 & 2,201 & 2,228 \\
\hline
\end{tabular}


do Espírito Santo ampliam o limite norte da distribuição geográfica dessa espécie, endêmica de Mata Atlântica, que alcança o Rio Grande do Sul no Brasil, Misiones na Argentina e leste do Paraguai. Trata-se de uma espécie semi-fossorial e muito pouco conhecida quanto à sua história natural (Oliveira \& Bonvicino 2006).

As maiores riqueza e diversidade encontradas no município de Viana podem estar associadas à heterogeneidade do hábitat, que é um dos principais fatores que determinam a ocorrência e distribuição de pequenos mamíferos não voadores (Pardini \& Umetsu 2006). Considerando que a região é formada por um mosaico de áreas de floresta e agrícolas, a diversidade relativamente alta encontrada no presente estudo deve estar associada ao fato da região apresentar níveis intermediários de distúrbio, que favorecem a diversidade (Vera y Conde \& Rocha 2006). De qualquer forma, os resultados evidenciam a importância dos fragmentos florestais desse município como depositários da biodiversidade da Mata Atlântica, principalmente por ser uma área periférica de uma grande região metropolitana e, portanto, sob forte pressão antrópica. Nesse sentido, o estabelecimento de uma unidade de conservação no município deve ser incentivado, uma vez que o mesmo não apresenta sequer uma área de proteção ambiental.

\section{Agradecimentos}

Aos João C. O. Rocha e Hygor M. Siqueira e moradores locais pela ajuda nos trabalhos de campo. Aos estagiários do Laboratório de Mastozoologia e Biogeografia pelo auxílio na taxidermia dos mamíferos coletados. Ao Vilacio Caldara Jr. por examinar os exemplares de Euryzygomatomys do Museu Nacional. Aos proprietários dos fragmentos florestais pelo consentimento nas capturas. Ao Instituto Brasileiro do Meio Ambiente e dos Recursos Naturais (IBAMA) por providenciar a licença de coleta dos mamíferos. À Coordenação de Aperfeiçoamento de Pessoal de Nível Superior (CAPES) e ao Critical Ecosystem Partnership Fund (CEPF) pelas bolsas concedidas respectivamente a ISP e ACCL.

\section{Referências Bibliográficas}

BALLESTRERO, H.L. 1951. Subsídios para o estudo da geografia e da história do município de Viana, Jabaeté, Espírito Santo. Escola Técnica de Vitória, Vitória, 225 p.

BONVICINO, C.R., LANGGUTH, A., LINDBERGH, S.M. \& PAULA, A.C. 1997. An elevational gradient study of small mammals at Caparaó National Park, southeastern Brazil. Mammalia. 61(4):547-560.

BONVICINO, C.R., LINDBERGH, S.M. \& MAROJA, L.S. 2002. Small non-flying mammals from conserved and altered areas of Atlantic Rain Forest and Cerrado: comments on their potential use for monitoring environment. Braz. J. Biol. 62(4):765-774.

CARÃO, L.M. 2008. Levantamento de mamíferos não-voadores da Reserva Biológica de Duas Bocas, Espírito Santo e comparação da eficiência dos métodos utilizados. Monografia, Faculdades Integradas de São Pedro, Vitória, $36 \mathrm{p}$.

DAJOZ, R. 1973. Ecologia geral. Universidade de São Paulo, São Paulo, $474 \mathrm{p}$.

DEAN, W. 1995. With broadax and firebrand: the destruction of the Brazilian Atlantic Forest. University of California Press, Berkeley, 285 p.
FALQUETO, A. \& FERREIRA, A.L. 2005. Reservatórios extra-humanos da Leishmânia e dinâmica de transmissão da infecção ao homem. In Dinâmica das doenças infecciosas e parasitárias (J.R. Coura, ed.). Guanabara Koogan, Rio de Janeiro, p. 739-752.

FEITOZA, L.R. 1999. Carta agroclimática do Espírito Santo. Secretaria de Estado da Agricultura \& Empresa Capixaba de Pesquisa Agropecuária, Espírito Santo.

FONSECA, G.A.B., HERRMANN, G., LEITE, Y.L.R., MITTERMEIER, R.A., RYLANDS, A.B. \& PATTON, J.L. 1996. Lista anotada dos mamíferos do Brasil. Occas. Pap. Conserv. Biol. 4:1-38.

Instituto de Pesquisa da Mata Atlântica - IPEMA. 2005. Conservação da Mata Atlântica no Estado do Espírito Santo: cobertura florestal e unidades de conservação. Conservação Internacional do Brasil \& IPEMA, Vitória, 112 p. (Programa Centros para a Conservação da Biodiversidade).

LAW, B.S. \& DICKMAN, C.R. 1998. The use of habitat mosaics by terrestrial vertebrate fauna: implications for conservation and management. Biodivers. Conserv. 7(3):323-333.

MITTERMEIER, R.A., MYERS, N. \& THOMSEN, J.T. 1998. Biodiversity hotspots and major tropical wilderness areas: approaches to setting conservation priorities. Conserv. Biol. 12(3):516-520.

MOREIRA, D.O., COUTINHO, B.R. \& MENDES, S.L. 2008. O status do conhecimento sobre a fauna de mamíferos do Espírito Santo baseado em registros de museus e literatura científica. Biota Neotrop. 8(2):http://www.biotaneotropica.org.br/v8n2/pt/abstract?thematicreview+bn02108022008 (último acesso em 31/08/2009).

MYERS, N., MITTERMEIER, R.A., MITTERMEIER, C.G., FONSECA, G.A.B. \& KENT, J. 2000. Biodiversity hotspots for conservation priorities. Nature. 403:853-858.

OLIVEIRA, J.A. \& BONVICINO, C.R. 2006. Ordem rodentia. In Mamíferos do Brasil (N.R. dos Reis, A.L. Peracchi, W.A. Pedro, I.P. de Lima, eds.) EDIFURB, Londrina, p. 347-406.

PARDINI, R. \& UMETSU, F. 2006. Non-volant small mammals from the Morro Grande Forest Reserve: distribution of species and diversity in an Atlantic Forest area. Biota Neotrop. 6(2): http://www.biotaneotropica. org.br/v6n2/pt/abstract?article+bn00606022006 (último acesso em 31/08/2009).

PARESQUE, R., SOUZA, W.P., MENDES, S.L. \& FAGUNDES, V. 2004. Composição cariotípica da fauna de roedores e marsupiais de duas áreas de Mata Atlântica do Espírito Santo, Brasil. Bol. Mus. Biol. Mello Leitão. 17(1):5-33.

PASSAMANI, M. 2003. O efeito da fragmentação da Mata Atlântica serrana sobre a fauna de pequenos mamíferos de Santa Teresa, Espírito Santo. Tese de Doutorado, Universidade Federal do Rio de Janeiro, Rio de Janeiro, $105 \mathrm{p}$.

QUINTELA, C.E. 1990. An S.O.S. for Brazil's beleaguered Atlantic Forest. Nat. Conserv. Mag. 40(2):14-19.

RUSCHI, A. 1978. Mamíferos e aves do Parque Nacional do Caparaó. Bol. Mus. Biol. Mello Leitão. 95:1-27.

VERA y CONDE, C.F. \& ROCHA, C.F.D. 2006. Habitat disturbance and small mammal richness and diversity in an Atlantic rainforest area in southeastern Brazil. Braz. J. Biol. 66(4):983-990.

WILSON, D.E. \& REEDER, D.M. (eds.) 2005. Mammal Species of the World: a Taxonomic and Geographic Reference. 3rd ed. Johns Hopkins University Press, Baltimore, 2142 p.

Recebido em 27/04/09 Versão reformulada recebida em 09/07/09 Publicado em 10/07/09 
Pinto, I.S. et al.

\begin{abstract}
ANEXO I
Anexo 1. Material testemunho dos mamíferos coletados em Viana, Espírito Santo, Brasil.

Annex 1. Voucher material of mammals trapped at Viana, Espírito Santo, Brazil.

Ordem Didelphimorphia

Caluromys philander (Total = 3): Fazenda Boa Baixa: UFES 81, Pimenta: UFES 82, UFES 83. Didelphis aurita $($ Total = 13): Coacas: LGA 1828, Fazenda Boa Baixa: UFES 689, UFES 690, UFES 691, Pimenta: UFES 692, UFES 693, UFES, 694, UFES 695, UFES 696, UFES 697, Ribeira: UFES 686, UFES 687, UFES 688. Gracilinanus microtarsus (Total = 2): Pimenta: UFES 698, Ribeira: UFES 699. Marmosa murina (Total = 25): Coacas: LGA 1822, LGA 1824, LGA 1831, UFES 709, UFES 708, UFES 710, Fazenda Boa Baixa: UFES 711, UFES 712, UFES 713, UFES 714, UFES 715, UFES 716, UFES 17, UFES 718, UFES 719, UFES 720, Formate: UFES 701, UFES 702, UFES 703, Pimenta: UFES 704, UFES 705, UFES 706, UFES 707, Povoação: UFES 823, Ribeira: UFES 700. Marmosops incanus (Total = 12): Formate: UFES 825, Pimenta: UFES 725, UFES 726, UFES 727, UFES 728, UFES 729, UFES 730, Povoação: UFES 824, Ribeira: UFES 721, UFES 722, UFES 723, UFES 724. Metachirus nudicaudatus (Total = 20): Coacas: LGA 1825, LGA 1830, Fazenda Boa Baixa: UFES 741, UFES 742, UFES 743, UFES 744, UFES 869, Pimenta: UFES 665, UFES 731, UFES 732, UFES 733, UFES 734, UFES 735, UFES 736, UFES 737, Povoação: UFES 826, UFES 827, Ribeira: UFES 738, UFES 739, UFES 740. Micoureus paraguayanus (Total = 17): Coacas: LGA 1823, LGA 1826, UFES 756, Fazenda Boa Baixa: UFES 670, UFES 745, Perobas: UFES 828, Pimenta: UFES 664, UFES 746, UFES 747, UFES 748, UFES 749, UFES 750, Ribeira: UFES 751, UFES 752, UFES 753, UFES 754, UFES 755. Monodelphis americana (Total = 2): Fazenda Boa Baixa: UFES 757, Povoação: UFES 829. Philander frenatus (Total = 2): Coacas: UFES 830, Povoação: UFES 831.
\end{abstract}

Ordem Rodentia

Akodon cursor $($ Total = 56): Coacas: LGA 1827, LGA 1829, LGA 1993, LGA 1995, LGA 1996, LGA 1997, LGA, 2002, LGA 2003, LGA 2004, LGA 2005,LGA 2009, LGA 2010, LGA 2011, LGA 2012, LGA 2013, LGA 2014, SLF 248, SLF 259, UFES 763, UFES 764, UFES 765, UFES 766, UFES 767, UFES 768, UFES 769, UFES 770, UFES 771, UFES 772, UFES 773, UFES 774, Fazenda Boa Baixa: UFES 675, UFES 676, UFES 677, UFES 678, UFES 679, UFES 680, UFES 681, UFES 775, UFES 776, UFES 777, UFES 778, UFES 779, UFES 780, UFES 781, UFES 782, UFES 784, UFES 785, UFES 786, UFES 787, UFES 788, UFES 789, Perobas: UFES 832, Povoação: UFES 833, UFES 834, Ribeira: UFES 790. Euryzygomatomys spinosus $($ Total = 1): Coacas: UFES 835. Guerlinguetus ingrami $($ Total = 6): Pimenta: UFES 666, UFES 758, UFES 759, UFES 760, UFES 761, Ribeira: UFES 762. Nectomys squamipes (Total = 26): Coacas: UFES 808, UFES 809, Fazenda Boa Baixa: UFES 685, UFES 805, UFES 806, UFES 807, Formate: LPC 988, UFES 800, UFES 801, UFES 802, UFES 803, UFES 804, Pimenta: UFES 668, UFES 672, UFES 791, UFES 792, UFES 793, UFES 794, UFES 795, UFES 796, UFES 797, UFES 798, UFES 799, Povoação: UFES 836, UFES 837, UFES 838. Oecomys catherinae (Total = 3): Fazenda Boa Baixa: UFES 810, UFES 811, UFES 812. Oligoryzomys sp. (Total =1): Coacas: UFES 839. Oxymycterus dasythrichus $($ Total = 1): Povoação: UFES 840. Phyllomys pattoni $($ Total = 1): Coacas: UFES 843. Rattus rattus $($ Total = 2): Coacas: UFES 841, Formate: UFES 842. Rhipidomys mastacalis $($ Total = 1): Ribeira: UFES 813. Trinomys paratus (Total = 17): Fazenda Boa Baixa: UFES 671, UFES 814, UFES 815, UFES 816, UFES 817, Formate: UFES 682, UFES 683, UFES 684, UFES 821, Perobas: UFES 844, Pimenta: LPC 978, UFES 669, UFES 818, UFES 819, UFES 820, Ribeira: UFES 673, UFES 674. 